\title{
Issues in implementing lung cancer screening in United States and Europe
}

\author{
Giuseppe Gorini, Giulia Carreras
}

Occupational \& Environmental Epidemiology Section, Oncologic Network, Prevention and Research Institute, Florence, Italy

Correspondence to: Giulia Carreras, PhD. Occupational \& Environmental Epidemiology Section, Oncologic Network, Prevention and Research Institute (ISPRO), via delle Oblate, 2 - 50141 Florence, Italy. Email: giulia.carreras@gmail.com.

Comment on: Zeliadt SB, Hoffman RM, Birkby G, et al. Challenges Implementing Lung Cancer Screening in Federally Qualified Health Centers. Am J Prev Med 2018;54:568-75.

Submitted Sep 21, 2018. Accepted for publication Oct 10, 2018.

doi: $10.21037 / \mathrm{atm} .2018 .10 .20$

View this article at: http://dx.doi.org/10.21037/atm.2018.10.20

\section{Introduction}

While recently the European lung cancer screening community published a position statement in order to promote the development in Europe of low-dose computed tomography (LDCT) lung cancer screening programmes (1), US Health Centers already began implementing lung cancer screening, and the article by Zeliadt et al. (2), reported the current state of the art of LDCT lung cancer screening in US Federally Qualified Health Centers. The authors focused on these types of clinics which predominantly serve low-socioeconomic populations, because of the suggestion of the Society of Behavioural Medicine which observed that disparities in lung cancer may be reduced implementing a high-quality lung cancer screening in high-risk populations (3).

Based on results of the National Lung Screening Trial (NLST) (4), LDCT screening in the US is generally covered by private or government insurances for the screeneligible population since 2015. However, the dissemination of LDCT screening has been slow: less than $5 \%$ of those eligible are screened (5). In the survey conducted by Zeliadt et al., only 3 out of 110 clinics reported screening more than 10 patients per month. Main barriers were the assessment of smoking history to identify eligible populations, potential gaps in smoking cessation resources, limited access to specialty providers to monitor abnormal findings and manage follow-up, lack of insurance coverage, and significant obstacles even to those with insurance, including out-of pocket costs for follow-up procedures (2).

Even though several organizations, such as the US
Preventive Service Task Force, recommended LDCT lung cancer screening (6), we have to take into account that the American Academy of Family Physicians reported concerns about the ability to replicate the NLST findings in community practice as a reason not to recommend screening (7).

\section{Suspicious nodules and incidental findings}

Both Europeans and Americans in LDCT lung cancer screening share the issue on management of suspicious nodules and costs of further evaluations in the followup. In the cost-effectiveness analysis of the NLST, the base-case scenario provided one follow-up screening with LDCT after a positive test (8). However, both suspicious and incidental findings require careful coordination with oncology, pulmonary, and cardiology departments, in order to determine whether findings require additional evaluations. In the implementation of lung cancer screening in the US Veterans Health Administration, 56\% of patients had one or more nodules needing to be tracked, and incidental findings, including emphysema, other pulmonary abnormalities and coronary artery calcification, were reported in $41 \%$ of patients (9). Moreover, as discussed in the article of Zeliadt (2), patients had to pay followup procedures. Thus, a challenge for the Europeans in the implementation of nation-wide lung cancer screening programmes could be to establish which or how many follow-up procedures will be covered by National Health Systems or insurances. 


\section{Cost-effectiveness}

Anyway, the biggest problem that both Europeans and Americans share is cost-effectiveness evaluation that does not allow, up to now, to conclude that LDCT is costeffective. In an era of constrained medical resources, the cost-effectiveness of this programme needs to be demonstrated. A recent review of 13 economic evaluations of lung cancer screening reported cost-effectiveness estimates with a large variation among studies (10). Studies using life-years gained as measure of outcome reported incremental cost-effectiveness ratios (ICERs) varying from US $\$ 18,452$ to US $\$ 66,480$ per life-year gained for repeated screening, whereas in studies using quality adjusted life years (QALYs) as outcome ICERs varied between US\$27,756 and US\$243,077 per QALY gained (10). The review concluded that cost-effectiveness of lung cancer screening is still unclear since there is too much uncertainty about critical parameters of delivering lung cancer screening to quantify the true cost of screening and determine whether LDCT screening is cost-effective (10). In fact, cost-effectiveness analyses were sensitive to many model parameters, including cost of LDCT of screening, the inclusion of a smoking cessation programme or referral system, mortality reductions or improvements to quality of life for participants without lung cancer, improved lung nodule management, more precise high-risk selection, more successful early stage treatments $(10,11)$. At least 10 costeffectiveness analyses on LDCT lung cancer screening have been conducted in the US (10). To our knowledge, only one study on healthcare costs was conducted in Europe, estimating the costs of the Danish LDCT lung cancer screening trial, and concluding that screening costs were higher compared with no screening (12).

\section{Smoking history ascertainment and smoking cessation}

Other issues that both Europeans and Americans share are on the ascertainment of smoking history for recruitment of ever smokers, and on the availability of smoking cessation resources embedded in lung cancer screening pathways (13). A special challenge of implementing an organized, population-based programme of screening for lung cancer, in comparison to breast, cervical, or colorectal cancer, is that the eligibility criteria are not only age and sex, but include characteristics not readily identifiable, including details about smoking history, years since quitting, and current smoking status $(14,15)$.

Regarding smoking cessation resources, Europeans are far behind Americans. Smoking cessation referral systems, such as the North American Quitline Consortium, (https://www. naquitline.org/) or digital media interventions, including web-based or text messaging-based interventions, and automated calling systems (https://smokefree.gov/), are well developed in US, whereas in Europe few Countries have already well-organized Quitlines, smoking cessation centers, and digital media interventions (http://www.tabac-infoservice.fr/, https://www.nhs.uk/smokefree). Moreover, only few European countries developed nation-wide campaigns linked to digital media with a clear behavioural target (making a serious quit attempt), such as the US "Tips from former smokers" (https://www.cdc.gov/tobacco/campaign/ tips/index.html). The impact of the campaign carried out in 2012 was studied in depth: quit attempts increased from $31 \%$ at baseline to $35 \%$ after the 3 -month campaign, with an estimated 1.64 million additional smokers who made a quit attempt, 220,000 remained abstinent at followup, and about 100,000 remained abstinent for more than one year. Recommendations by non-smokers to quit increased from $3 \%$ at baseline to $5 \%$ at follow-up, and the prevalence of people talking about the dangers of smoking increased from $32 \%$ to $35 \%$, resulting in an estimated 4.7 million additional non-smokers recommending cessation services and more than 6 million talking about the dangers of smoking. Tips saved about 179,099 QALYs and prevented 17,109 premature deaths in the US. With the campaign cost about $\$ 48$ million, Tips spent approximately $\$ 480$ per quitter, $\$ 2,819$ per premature death averted, $\$ 393$ per LY saved, and \$268 per QALY gained. Thus, the Tips media campaign was effective at increasing population-level quit attempts, at reducing smoking-attributable morbidity and mortality, and finally was a highly cost-effective mass media intervention $(16,17)$. To our knowledge, only in UK since 2012, in the Netherlands since 2014, and in France since 2016, Stoptober campaigns ("Mois sans tabac" in French) were introduced to promote quitting smoking for 28 days in a specific month (i.e., October). These campaigns used a web-based approach and the same behavioural target (making a serious quit attempt) of the US Tips campaign (https://www.nhs.uk/oneyou/stoptober/home, https:// stoptober.nl/, http://mois-sans-tabac.tabac-info-service.fr/). The UK 2012 Stoptober campaign was evaluated: more people tried to quit in October in 2012 compared to those trying in the period 2007-2011 (odds ratio $=1.79 ; \mathrm{P}<0.05$ ). Stoptober campaign in UK determined 350,000 quit 
attempts, and saved 10,400 discounted life years (DLYs) at about $£ 560$ per DLY (18).

\section{A new strategy for lung cancer screening recruitment and smoking cessation: the Opt-out strategy}

More importantly, Europe does need a new approach to smoking cessation, as US and Canada already developed in recent years. In the US, the Joint Commission's new Tobacco Use Performance Measure Set took effect in 2012. These hospital quality measures require hospitals to offer tobacco-cessation counseling and pharmacotherapy to all hospitalized smokers, to follow-up smokers within one month after hospital discharge, and to provide or refer smokers to treatment resources after discharge (19). In Canada a similar approach was developed since 2010 with the Ottawa Model for Smoking Cessation (20). These strategies are required in order to offer smoking cessation resources and referral systems within lung cancer screening programmes. Similar approaches are not yet developed in Europe (13). In fact, recently, the UK Royal College of Physicians recognized that ascertainment and treatment of smokers using National Health System (NHS) services in UK use an opt-in design, and is not well embedded in patient pathways or disease treatment guidelines (21). A rational approach in Europe could be to adopt an opout strategy for smoking cessation in primary care and in health services in order to ensure that ascertainment and treatment of smokers becomes a core NHS activity. In Europe, training in smoking cessation interventions for healthcare professionals is inadequate (21). Smoking is most commonly taught as part of a more general topic such as health promotion. Training tools, such as those provided by the Canadian Ottawa Model for Smoking Cessation (20,22), or by the UK National Centre for Smoking Cessation and Training (http://elearning.ncsct.co.uk/vba-stage_1), are not available for most European health professionals. Thus, in Europe training in smoking cessation interventions has to be introduced into all undergraduate and postgraduate healthcare professional curricula to ensure that all new health professionals are appropriately trained.

The introduction of smoking cessation training for health professionals and of an op-out strategy in smoking cessation should improve data collection needed for recruitment of patients in lung cancer screening programmes (smoking history, years since quitting), and could allow to have already available smoking cessation interventions to be embedded within lung cancer screening programmes.

\section{Conclusions}

In conclusion, while in Europe smoking cessation interventions are not yet well developed within health services, and lung cancer screening programmes have not yet started, in US, where a systematic approach to smoking cessation is being developed, the implementation of lung cancer screening is slow and showed problems linked above all to the management and cost of follow-up procedures. More importantly, for both Europe and US, there are not yet definite results on cost-effectiveness studies. Given that data from US showed that implementing a lung cancer screening programme is a challenging endeavour even when smoking cessation resources are available, it is important for European countries to await for new cost-effectiveness evaluations conducted in Europe to confirm the costeffectiveness of such a complex intervention, and, in the meantime, to develop smoking cessation referral systems within health services in order to adequately support smoking cessation.

\section{Acknowledgements}

None.

\section{Footnote}

Conflicts of Interest: The authors have no conflicts of interest to declare.

\section{References}

1. Oudkerk M, Devaraj A, Vliegenthart R, et al. European position statement on lung cancer screening. Lancet Oncol 2017;18:e754-66.

2. Zeliadt SB, Hoffman RM, Birkby G, et al. Challenges Implementing Lung Cancer Screening in Federally Qualified Health Centers. Am J Prev Med 2018;54:568-75.

3. Watson KS, Blok AC, Buscemi J, et al. Society of Behavioral Medicine supports implementation of high quality lung cancer screening in high-risk populations. Transl Behav Med 2016;6:669-71.

4. Aberle DR, Adams AM, Berg CD, et al. Reduced lungcancer mortality with low-dose computed tomographic screening. N Engl J Med 2011;365:395-409.

5. Pinsky PF. Does the evidence support the implementation 
of lung cancer screening with low-dose computed tomography? Expert Rev Respir Med 2018;12:257-260.

6. Moyer VA, U.S. Preventive Services Task Force.. Screening for lung cancer: U.S. Preventive Services Task Force recommendation statement. Ann Intern Med 2014;160:330-8.

7. American Academy of Family Physicians. Clinical preventive service recommendation: lung cancer. Available online: https://www.aafp.org/patient-care/ clinical-recommendations/all/lung-cancer.html. Accessed September 11, 2018.

8. Black WC, Gareen IF, Soneji SS, et al. Cost-effectiveness of CT screening in the national lung screening trial. $\mathrm{N}$ Engl J Med 2014;371:1793-802.

9. Kinsinger LS, Anderson C, Kim J, et al. Implementation of Lung Cancer Screening in the Veterans Health Administration. JAMA Intern Med 2017;177:399-406.

10. Raymakers AJN, Mayo J, Lam S, et al. Cost-Effectiveness Analyses of Lung Cancer Screening Strategies Using LowDose Computed Tomography: a Systematic Review. Appl Health Econ Health Policy 2016;14:409-18.

11. Cressman S, Peacock SJ, Tammemägi MC, et al. The Cost-Effectiveness of High-Risk Lung Cancer Screening and Drivers of Program Efficiency. J Thorac Oncol 2017;12:1210-22.

12. Rasmussen JF, Siersma V, Pedersen JH, et al. Healthcare costs in the Danish randomised controlled lung cancer CT-screening trial: a registry study. Lung Cancer 2014;83:347-55.

13. Carreras G, Gorini G. Challenges of quitting smoking and lung cancer screening. Ann Transl Med 2017;5:488.

14. van der Aalst CM, Ten Haaf K, de Koning HJ. Lung

Cite this article as: Gorini G, Carreras G. Issues in implementing lung cancer screening in United States and Europe. Ann Transl Med 2018;6(Suppl 1):S54. doi: 10.21037/ atm.2018.10.20 cancer screening: latest developments and unanswered questions. Lancet Respir Med 2016;4:749-61.

15. Field JK, Duffy SW, Devaraj A, et al. Implementation planning for lung cancer screening: five major challenges. Lancet Respir Med 2016;4:685-7.

16. McAfee T, Davis KC, Alexander RL Jr, et al. Effect of the first federally funded US antismoking national media campaign. Lancet 2013;382:2003-11.

17. Xu X, Alexander RL Jr, Simpson SA, et al. A costeffectiveness analysis of the first federally funded antismoking campaign. Am J Prev Med 2015;48:318-25.

18. Brown J, Kotz D, Michie S, et al. How effective and cost-effective was the national mass media smoking cessation campaign 'Stoptober'? Drug Alcohol Depend 2014;135:52-8.

19. Fiore MC, Goplerud E, Schroeder SA. The joint commission's new tobacco-cessation measures-will hospitals do the right thing? N Engl J Med 2012;366:1172-4.

20. Reid RD, Mullen KA, Slovinec D'Angelo ME, et al. Smoking cessation for hospitalized smokers: an evaluation of the "Ottawa Model". Nicotine Tob Res 2010;12:11-8.

21. Royal College of Physicians. Hiding in plain sight: treating tobacco dependency in the NHS. London: RCP, 2018. Available online: https://www.rcplondon.ac.uk/projects/ outputs/hiding-plain-sight-treating-tobacco-dependencynhs. Accessed July 23, 2018.

22. Mullen KA, Manuel DG, Hawken SJ, et al. Effectiveness of a hospital-initiated smoking cessation programme: 2 -year health and healthcare outcomes. Tob Control 2017;26:293-9. 